A) Check for updates

Cite this: Nanoscale, 2021, 13, 4644

Received 1st December 2020,

Accepted 6th January 2021

DOI: $10.1039 /$ dOnr08537e

rsc.li/nanoscale

\section{Influence of interaction between surface-modified magnetic nanoparticles with infectious biofilm components in artificial channel digging and biofilm eradication by antibiotics in vitro and in vivo†}

\author{
Kecheng Quan, ${ }^{a, b}$ Guimei Jiang, ${ }^{b, c}$ Jian Liu, (D) ${ }^{c}$ Zexin Zhang, (D) *a Yijin Ren, ${ }^{d}$ \\ Henk J. Busscher, ${ }^{* \mathrm{~b}}$ Henny C. van der Mei (D) ${ }^{\mathrm{b}}$ and Brandon W. Peterson (DD ${ }^{* b}$
}

\begin{abstract}
Magnetic targeting of antimicrobial-loaded magnetic nanoparticles to micrometer-sized infectious biofilms is challenging. Bacterial biofilms possess water channels that facilitate transport of nutrient and metabolic waste products, but are insufficient to allow deep penetration of antimicrobials and bacterial killing. Artificial channel digging in infectious biofilms involves magnetically propelling nanoparticles through a biofilm to dig additional channels to enhance antimicrobial penetration. This does not require precise targeting. However, it is not known whether interaction of magnetic nanoparticles with biofilm components impacts the efficacy of antibiotics after artificial channel digging. Here, we functionalized magnetic-iron-oxide-nanoparticles (MIONPs) with polydopamine (PDA) to modify their interaction with staphylococcal pathogens and extracellular-polymeric-substances (EPS) and relate the interaction with in vitro biofilm eradication by gentamicin after magnetic channel digging. PDA-modified MIONPs had less negative zeta potentials than unmodified MIONPs due to the presence of amino groups and accordingly more interaction with negatively charged staphylococcal cell surfaces than unmodified MIONPs. Neither unmodified nor PDA-modified MIONPs interacted with EPS. Concurrently, use of non-interacting unmodified MIONPs for artificial channel digging in in vitro grown staphylococcal biofilms enhanced the efficacy of gentamicin more than the use of interacting, PDA-modified MIONPs. In vivo experiments in mice using a sub-cutaneous infection model confirmed that non-interacting, unmodified MIONPs enhanced eradication by gentamicin of Staphylococcus aureus Xen36 biofilms about 10 fold. Combined with the high biocompatibility of magnetic nanoparticles, these results form an important step in understanding the mechanism of artificial channel digging in infectious biofilms for enhancing antibiotic efficacy in hard-to-treat infectious biofilms in patients.
\end{abstract}

\section{Introduction}

${ }^{a}$ College of Chemistry, Chemical Engineering and Materials Science, Soochow University, Suzhou 215123, P.R. China. E-mail: zhangzx@suda.edu.cn; Tel: +8651269155295

${ }^{b}$ University of Groningen and University Medical Center Groningen, Department of Biomedical Engineering, 9713 AV Groningen, The Netherlands.

E-mail:h.j.busscher@umcg.nl, b.w.peterson@umcg.nl; Tel: +31503616094, $+31503616110$

${ }^{c}$ Institute of Functional Nano \& Soft Materials (FUNSOM), Jiangsu Key Laboratory for Carbon-Based Functional Materials \& Devices, Collaborative Innovation Center of Suzhou Nano Science and Technology, Soochow University, Suzhou 215123, P. R. China

${ }^{d}$ University of Groningen and University Medical Center Groningen, Department of Orthodontics, Hanzeplein 1, 9713 GZ Groningen, The Netherlands

$\dagger$ Electronic supplementary information (ESI) available. See DOI: 10.1039/ donr08537e
The treatment of infectious biofilms is extremely challenging, despite the availability of antibiotics, ${ }^{1}$ newly developed antimicrobial nanoparticles to enhance antibiotic activity ${ }^{2,3}$ and facilitating photothermal killing. ${ }^{4,5}$ Magnetic targeting of drug-loaded magnetic nanoparticles has been described as promising in cancer therapy ${ }^{6-8}$ and eradication of infectious bacterial biofilms. ${ }^{9,10}$ However, magnetic targeting of drugloaded nanocarriers to a micrometer-sized infectious biofilm is highly challenging compared with the targeting of centimeter-sized tumors ${ }^{11}$ and effective bacterial killing over the depth of a biofilm by antimicrobial-loaded magnetic nanoparticles can only be achieved if the nanoparticles are well-distributed over the depth of a biofilm. This precise targeting of a 
biofilm as a whole requires accurate timing and magnetic field control. $^{12}$ As a consequence, this 3D challenge may well impede the clinical translation of the use of antimicrobialloaded magnetic nanoparticles for bacterial infection control based on targeting. ${ }^{13,14}$

Biofilms consist for the major part of water confined in channels and pores within a matrix of extracellular polymeric substances (EPS) that serve to transport and store nutrients and metabolic waste products, respectively. ${ }^{15}$ Naturally occurring channels in bacterial biofilms are not sufficient however, to allow deep penetration of antimicrobials, attesting to the protective function of the biofilm-mode of growth. Natural water channels are created in Bacillus thuringiensis biofilms by flagella-propelled bacterial movement. ${ }^{16}$ Inspired by the natural ability of motile swimmers to dig channels in a biofilm, infectious Staphylococcus aureus biofilms have been irrigated with bacterial swimmers to dig additional channels to facilitate deep penetration of antibiotics, but in vivo benefits have not yet been demonstrated. For clinical translation, this would involve the deliberate introduction of a bacterial strain in the body of an already infected patient, which is highly undesirable. Moreover, bacterially-dug channels are quite large and therewith transient due to the visco-elastic nature of biofilm, causing channel collapse. Both problems can be overcome, by magnetically propelling nanoparticles through a biofilm. Magnetic propulsion of simple, unmodified magnetic iron oxide nanoparticles through an $S$. aureus biofilm created additional artificial channels that increased in vitro killing of biofilm inhabitants by gentamicin $4-6$ fold. ${ }^{17}$

The aim of this work is, to determine whether interaction of magnetic iron oxide nanoparticles (MIONPs) with biofilm components influences the efficacy of antibiotic treatment of an $S$. aureus biofilm after artificial channel digging by magnetically propelled nanoparticles in vitro. Two $S$. aureus strains will be used, with different ability to produce EPS. ${ }^{18,19}$ MIONPs will be made adhesive, by polymerizing dopamine $^{20-22}$ on MIONP surfaces (see Fig. 1a). Interaction between MIONPs and PDA-modified MIONPs with staphylococci and EPS will be evaluated and related with enhancement of gentamicin efficacy after additional artificial channel digging in vitro. In vivo benefits of artificial channel digging on eradication by gentamicin of an infectious $S$. aureus Xen36 biofilm will be evaluated in a murine model.

\section{Experimental section}

\section{Materials}

Gentamicin, kanamycin, and iron(III) chloride $\left(\mathrm{FeCl}_{3} \cdot 6 \mathrm{H}_{2} \mathrm{O}\right)$ were purchased from Sigma-Aldrich (Zwijndrecht, The Netherlands). Trisodium citrate dehydrate $\left(\mathrm{C}_{6} \mathrm{H}_{5} \mathrm{O}_{7} \mathrm{Na}_{3} \cdot 2 \mathrm{H}_{2} \mathrm{O}\right)$, urea, polyacrylamide (PAM, $300 \mathrm{kDa}$ ), dopamine hydrochloride, formaldehyde, glutaraldehyde $(50 \%, \mathrm{v} / \mathrm{v})$, dimethylsulfoxide (DMSO) and ethanol were purchased from Sinopharm Chemical Reagent Co. (China). Sodium chloride ( $\mathrm{NaCl})$, monopotassium phosphate $\left(\mathrm{KH}_{2} \mathrm{PO}_{4}\right)$, dipotassium phosphate $\left(\mathrm{K}_{2} \mathrm{HPO}_{4}\right)$, penicil- (a)
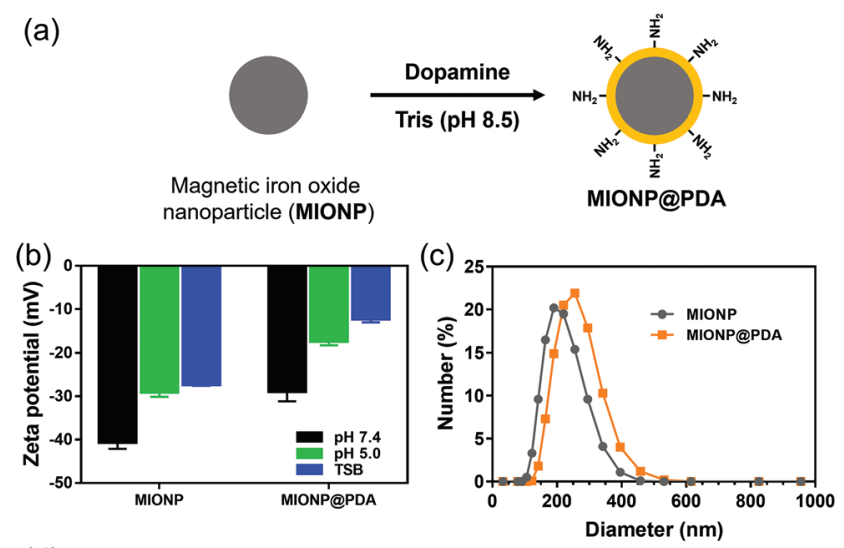

(d)
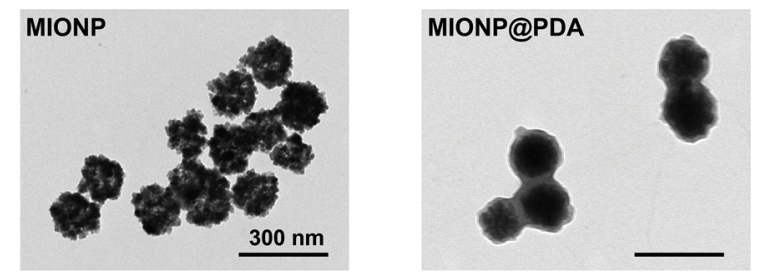

Fig. 1 Preparation of unmodified (MIONP) and PDA-modified (MIONP(APDA) magnetic iron-oxide nanoparticles, their zeta potential and size. (a) Dopamine was polymerized on the surface of MIONPs in a Tris ( $\mathrm{pH}$ 8.5) solution at room temperature and attached through hydrogen bonding to the MIONP surface with phenolic hydroxyl groups (not shown) present in DPA. ${ }^{35,36}$ (b) Zeta potentials of magnetic nanoparticles suspended in phosphate buffered saline (PBS, pH 7.4 and 5.0) and tryptone soy broth (TSB). Zeta potentials are expressed as means \pm standard deviations over three separate experiments with different batches of nanoparticles. (c) Diameter distribution of magnetic nanoparticles suspended in PBS. (d) TEM micrographs of unmodified and PDA-modified magnetic nanoparticles.

lin and streptomycin were purchased from Aladdin (China). All chemicals were used as received.

\section{Preparation and characterizations of MIONPs and MIONP@PDA}

Magnetic nanoparticles (MIONPs) were synthesized according to a previously reported method. ${ }^{23}$ Briefly, $2 \mathrm{mmol} \mathrm{FeCl}_{3} \cdot 6 \mathrm{H}_{2} \mathrm{O}$ $(0.54 \mathrm{~g}), 4 \mathrm{mmol} \mathrm{C}_{6} \mathrm{H}_{5} \mathrm{O}_{7} \mathrm{Na}_{3} \cdot 2 \mathrm{H}_{2} \mathrm{O}(1.18 \mathrm{~g})$ and $6 \mathrm{mmol}$ urea $(0.36 \mathrm{~g})$ were dissolved in $40 \mathrm{~mL}$ of distilled water, and $0.3 \mathrm{~g}$ PAM (300 kDa) was added and stirred for $60 \mathrm{~min}$. Subsequently, the solution was heated to $200{ }^{\circ} \mathrm{C}$ for $12 \mathrm{~h}$ in a $100 \mathrm{~mL}$ Teflon-lined stainless-steel autoclave. After cooling down to room temperature (RT), the black nanoparticles were magnetically separated and washed with distilled water and ethanol each for 3 times and MIONPs collected by magnetic separation. Finally, the black powder was dried in vacuum overnight. Polydopamine-modified MIONPs (MIONP@PDA) were synthesized by dispersing $90 \mathrm{mg}$ of the MIONPs obtained and $30 \mathrm{mg}$ dopamine hydrochloride in $30 \mathrm{~mL}$ Tris $(\mathrm{pH} 8.5)$ solution. This suspension was stirred in a sonication bath (Transonic TP 690, ELMA, Germany, $160 \mathrm{~W}, 35 \mathrm{kHz}$ ) for $4 \mathrm{~h}$ at RT. The nanoparticles were washed 3 times by distilled water and dried in vacuum overnight. For further use, magnetic nanoparticles were suspended in phosphate buffered saline 
(PBS, $5 \mathrm{mM} \mathrm{K}_{2} \mathrm{HPO}_{4}, 5 \mathrm{mM} \mathrm{KH} \mathrm{PO}_{4}, 150 \mathrm{mM} \mathrm{NaCl}, \mathrm{pH}$ 7.4) or tryptone soy broth (TSB, OXOID, UK) by sonicating for $30 \mathrm{~min}$ at RT and stored at RT.

Zeta potentials were measured by suspending the magnetic nanoparticles in PBS ( $\mathrm{pH} 7.4$ and $\mathrm{pH}$ 5.0) and TSB using a Malvern NanoSizer ZS2000 (UK). Hydrodynamic diameters were measured by dynamic light scattering (DLS, Malvern ZetaSizer ZS2000, UK) in PBS. Transmission electron microscopy (TEM, G-120, Hitachi, Japan) was used to observe the size and shape of the magnetic nanoparticles. To this end, magnetic nanoparticles suspended in PBS were deposited on a carbon coated copper grid and oven-dried before imaging.

\section{Bacterial strains, growth conditions, and harvesting}

EPS-producing $S$. aureus Xen36 ${ }^{18,19}$ was grown from frozen stock solutions ( $7 \mathrm{v} / \mathrm{v} \% \mathrm{DMSO} / \mathrm{TSB}$ in the presence of kanamycin $\left.\left(10 \mu \mathrm{g} \mathrm{mL}^{-1}\right)\right)$ on TSB supplemented with kanamycin agar plate at $37{ }^{\circ} \mathrm{C}$ for $24 \mathrm{~h}$. A single bacterial colony was transferred into $10 \mathrm{~mL}$ of TSB with kanamycin and incubated for $24 \mathrm{~h}$ at $37^{\circ} \mathrm{C}$ as a preculture. The preculture was poured into $200 \mathrm{~mL}$ TSB with kanamycin and followed with another $16 \mathrm{~h}$ incubation at $37^{\circ} \mathrm{C}$ as a main culture. Bacteria were harvested by centrifugation $\left(5000 \mathrm{~g}, 5 \mathrm{~min}, 10^{\circ} \mathrm{C}\right.$ ) and washed twice with sterile PBS. The bacterial suspension was sonicated 3 times each for $30 \mathrm{~s}$ with $15 \mathrm{~s}$ intervals between each cycle in an ice bath to obtain single bacteria in the suspension. Bacterial concentration was calculated by measuring the UV absorbance of the suspension by a photo-spectrophotometer (Shimadzu, Japan) at $650 \mathrm{~nm}$ according to a standard curve between the absorbance value and bacterial concentration. Non-EPS producing $S$. aureus $5298^{18,19}$ was cultured in the same way as described above, but in TSB without the presence of kanamycin.

\section{Minimal inhibitory and bactericidal concentration (MIC/MBC)}

To evaluate the MICs and MBCs of S. aureus Xen36 and S. aureus 5298, $100 \mu \mathrm{L}$ of gentamicin $\left(100 \mu \mathrm{g} \mathrm{mL}{ }^{-1}\right)$, MIONPs or polydopamine-modified MIONPs $\left(2 \mathrm{mg} \mathrm{mL}^{-1}\right)$ in PBS were added into a 96-wells plate containing $100 \mu \mathrm{L}$ of TSB per well. Solutions were serially diluted 2 -fold and followed with the addition of $10 \mu \mathrm{L}$ of bacterial suspension $\left(10^{5} \mathrm{CFU} \mathrm{mL} \mathrm{mL}^{-1}\right)$ to each well. The mixture was incubated for $24 \mathrm{~h}$ at $37^{\circ} \mathrm{C}$ and the MIC value was taken as the lowest concentration at which bacterial growth was absent by visual observation. Then, $10 \mu \mathrm{L}$ of suspension was taken out from each well not demonstrating visual growth and dropped on an agar plate. After $24 \mathrm{~h}$ incubation at $37^{\circ} \mathrm{C}$, the lowest concentration at which no colonies were formed on the agar plate was taken as the MBC value. The experiment was performed in triplicate with separately cultured bacteria.

\section{Interaction of MIONPs and polydopamine-modified MIONPS with bacteria}

In order to investigate the interaction between MIONPs or polydopamine-modified MIONPs with staphylococci, $150 \mu \mathrm{L}$ of bacterial suspension $\left(2 \times 10^{8} \mathrm{CFU} \mathrm{mL}^{-1}\right)$ in PBS was mixed with $150 \mu \mathrm{L}$ of magnetic nanoparticles $\left(1 \mathrm{mg} \mathrm{mL}^{-1}\right)$ in PBS for
5 min in a 96-wells plate. Then a NdFeB magnet $(1 \mathrm{~mm}$ thickness and $10 \mathrm{~mm}$ in diameter with $1.2 \mathrm{~T}$ residual magnetism) was held underneath a well for $5 \mathrm{~min}$ to magnetically separate suspended magnetic nanoparticles from bacteria. After the separation, $50 \mu \mathrm{L}$ of the bacterial suspension was taken and the percentage of the number of bacteria remaining in suspension (i.e. not interacting with magnetic nanoparticles) was determined by CFU enumeration. Bacteria in PBS without magnetic nanoparticles served as a control. The experiments were done 3 times with separate bacterial cultures grown on different days.

For SEM observation of the interaction between MIONPs and polydopamine-modified MIONPs and staphylococci, $10 \mu \mathrm{L}$ of a mixed suspension of bacteria and magnetic nanoparticles was deposited on a silicon wafer. After drying at RT for $24 \mathrm{~h}$, the silicon wafers were immersed in $2.5 \%$ glutaraldehyde for $30 \mathrm{~min}$ at $4{ }^{\circ} \mathrm{C}$ for fixation. Next, the silicon wafers with deposited bacteria and magnetic nanoparticles were immersed in a 50, 60, 70, 80, 90, 100\% ethanol series for 10 min per concentration for bacterial dehydration. Finally, samples were dried in vacuum overnight before SEM (S-4700, Hitachi, Japan) observation.

\section{Interaction of MIONPs and polydopamine-modified MIONPs with EPS}

In order to investigate the interaction between MIONPs or polydopamine-modified MIONPs with EPS, EPS was first collected from $S$. aureus Xen36 biofilm, as previously described. ${ }^{24}$ Briefly, $20 \mathrm{~mL}$ of an $S$. aureus Xen36 suspension $\left(1 \times 10^{8} \mathrm{CFU}\right.$ $\mathrm{mL}^{-1}$ ) in PBS was added into a $1 \mathrm{~L}$ conical flask and left for $2 \mathrm{~h}$ at RT to allow bacterial adhesion. The liquid was removed and the flask was washed 3 times with PBS to remove nonadhered bacteria. Then, $400 \mathrm{~mL}$ TSB was added and the flask was put in an incubator for 6 days at $37^{\circ} \mathrm{C}$. Growth medium was refreshed every two days. The biofilm "sludge" was harvested by centrifugation $\left(5000 \mathrm{~g}, 5 \mathrm{~min}, 10^{\circ} \mathrm{C}\right)$, suspended in $100 \mathrm{~mL}$ PBS with $600 \mu \mathrm{L}$ formaldehyde (36.5\%) added to prevent bacterial lysis during following steps. Next, the suspension was shaken (100 rpm) at RT and after $1 \mathrm{~h}, 40 \mathrm{~mL} \mathrm{NaOH}(1$ $\mathrm{M})$ was added to extract the EPS. After shaking for another $3 \mathrm{~h}$, bacteria were collected by centrifugation $\left(5000 \mathrm{~g}, 5 \mathrm{~min}, 10^{\circ} \mathrm{C}\right.$ ) and the EPS containing supernatant was stored at $4{ }^{\circ} \mathrm{C}$ for further experiments.

The interaction between magnetic nanoparticles with EPS was evaluated by mixing $0.95 \mathrm{~mL}$ of the EPS containing supernatant with $0.05 \mathrm{~mL}$ MIONPs $\left(10 \mathrm{mg} \mathrm{mL}^{-1}\right)$ for $5 \mathrm{~min}$ at RT. Then, magnetic nanoparticles were magnetically separated from the mixture during $10 \mathrm{~min}$ and the weight of the remaining EPS was measured after freeze-drying. Interaction between magnetic nanoparticles and EPS was quantified as

$$
\operatorname{EPS}(\%)=W_{\mathrm{NP}} / W_{\mathrm{PBS}} \times 100 \%
$$

where $W_{\mathrm{PBS}}$ and $W_{\mathrm{NP}}$ represents the EPS weight remaining after mixing with PBS or a magnetic nanoparticle (NP) suspension, respectively. 


\section{In vitro biofilm growth, artificial channel digging and antibiotic killing}

For biofilm growth, $500 \mu \mathrm{L}$ of a staphylococcal suspension $(1 \times$

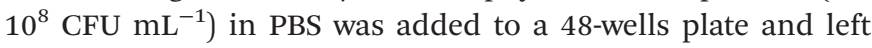
for $2 \mathrm{~h}$ at RT to allow bacterial adhesion, after which liquid was removed and the well was washed 3 times with PBS to remove the non-adhering bacteria. Next, $1 \mathrm{~mL}$ of TSB was added and the 48-well plate was put in an incubator for $24 \mathrm{~h}$ at $37^{\circ} \mathrm{C}$. After $24 \mathrm{~h}$, growth medium was removed and the biofilm was washed once with PBS. Then, $500 \mu \mathrm{L}$ of PBS containing magnetic nanoparticles $\left(0.5 \mathrm{mg} \mathrm{mL}^{-1}\right)$ was added, with or without gentamicin $\left(1.25 \mathrm{mg} \mathrm{mL}{ }^{-1}\right)$ supplementation, followed by 5 min magnetically-propelled movement of magnetic nanoparticles parallel and perpendicular with respect to the bottom of the wells. After $3 \mathrm{~h}$ incubation at $37{ }^{\circ} \mathrm{C}$, the nanoparticle suspension was removed and the biofilm was re-suspended in $1 \mathrm{~mL}$ of PBS, serially diluted and plated on TSB agar plates. After $24 \mathrm{~h}$, the number of CFUs were enumerated and the bacterial killing efficacy of gentamicin in biofilms with or without artificial channel digging using magnetically propelled nanoparticles was calculated. Biofilms treated by PBS alone served as a control. The experiments were done 3 times with separate bacterial cultures grown on different days.

In addition, biofilms before and after magnetically-propelled movement of different magnetic nanoparticles were visualized using confocal laser scanning microscopy (CLSM) after SYTO9 staining (Thermo Fisher Scientific, USA) for 15 min at RT. After staining, biofilms were washed once with PBS and imaged using a Leica TCS SP2 (Leica, Wetzlar, Germany) with an HCX APO L40×/0.80 W U-V-1 objective. An argon ion laser at $488 \mathrm{~nm}$ was used to excite the SYTO9 and fluorescence was collected at 500-540 nm. CLSM images were acquired using Leica software, version 2.0. Overlayer images of biofilms were made by stacking images using Image (NIH Research Services Branch, USA), while transverse, cross-sectional views were digitally generated by digital slicing of $5 \mu \mathrm{m}$ thick biofilm sections.

\section{In vitro biocompatibility}

Biocompatibility of MIONPs or polydopamine-modified MIONPs with L929 fibroblasts from murine connective tissue was evaluated using a metabolic activity assay. ${ }^{25}$ Briefly, fibroblasts were thawed from frozen stock and suspended in Dulbecco's Eagle's medium (DMEM-HG, ThermoFisher Scientific, USA), supplemented with $10 \%$ fetal bovine serum (FBS, Invitrogen, USA), $100 \mathrm{U} \mathrm{mL}^{-1}$ penicillin and $100 \mu \mathrm{g} \mathrm{mL}$ streptomycin. Suspended cells were incubated in tissue culture flasks filled with at $37{ }^{\circ} \mathrm{C}$ under $5 \% \mathrm{CO}_{2}$ and medium was refreshed daily. After five days confluency was reached and cells were harvested by trypsinization using trypsin-EDTA and seeded to the bottom of a 96-wells plate at a density of $5 \times 10^{3}$ cells per well followed by incubation for $24 \mathrm{~h}$ at $37^{\circ} \mathrm{C}$ under $5 \% \mathrm{CO}_{2}$. Then, $100 \mu \mathrm{L}$ of magnetic nanoparticles at different concentrations $\left(0.25,0.5\right.$ and $\left.1 \mathrm{mg} \mathrm{mL}^{-1}\right)$ suspended in cell growth medium, were added and incubation continued for another $24 \mathrm{~h}$. After $24 \mathrm{~h}, 50 \mu \mathrm{L}$ of XTT (Applichem, Germany) reagent solution combined with activation solution (PMS (n-methyl dibenzopyrazine methyl sulfate), Sigma-Aldrich, USA) was added. After $4 \mathrm{~h}$ at $37^{\circ} \mathrm{C}$, suspensions were centrifuged at $10000 \mathrm{~g}$ for $2 \mathrm{~min}$ and the absorbance of the supernatant was measured using a spectrophotometer at 485 and $690 \mathrm{~nm}$. Fibroblasts exposed to growth medium in absence of magnetic nanoparticles were taken as a blank control. Percentage viability of the fibroblasts after exposure to magnetic nanoparticles was calculated with respect to blank control according to

$$
\begin{aligned}
\operatorname{Viability}(\%)= & \left(\operatorname{AbS}_{\mathrm{NP} \text { at } 485 \mathrm{~nm}}-\operatorname{AbS}_{\mathrm{NP} \text { at } 690 \mathrm{~nm}}\right) / \\
& \left(\mathrm{AbS}_{\text {blank control at } 485 \mathrm{~nm}}-\mathrm{AbS}_{\text {blank control at } 690 \mathrm{~nm}}\right) \\
& \times 100 \%
\end{aligned}
$$

where $\mathrm{AbS}_{\mathrm{NP}}$ at $485 \mathrm{~nm}, \mathrm{AbS}_{\mathrm{NP}}$ at $690 \mathrm{~nm}, \mathrm{AbS}_{\text {blank control at } 485 \mathrm{~nm}}$ and $\mathrm{Abs}_{\text {blank control at } 690 \mathrm{~nm}}$ represent the absorbances of the cell suspensions with (NP) or without magnetic nanoparticles (blank control) at 485 and $690 \mathrm{~nm}$.

Biocompatibility of MIONPs or polydopamine-modified MIONPs with red blood cells (RBCs) was evaluated using a hemolysis assay. ${ }^{26,27}$ Anticoagulant citrate dextrose supplemented whole blood from mice $(2 \mathrm{~mL}$ ) was added to $4 \mathrm{~mL}$ PBS and centrifuged at $500 \mathrm{~g}$ for $10 \mathrm{~min}$ at $4{ }^{\circ} \mathrm{C}$ to collect RBCs. Centrifugation was repeated two more times to purify the RBCs suspension and RBCs collected were suspended in $10 \mathrm{~mL}$ PBS. Next, $0.1 \mathrm{~mL}$ of a MIONP suspension $(0.5,1$ and $2 \mathrm{mg} \mathrm{mL}{ }^{-1}$ ) was mixed with $0.1 \mathrm{~mL}$ of RBCs in PBS and incubated under shaking (190 rpm $\mathrm{min}^{-1}$ ) for $3 \mathrm{~h}$ at $37{ }^{\circ} \mathrm{C}$. After incubation, the mixture was centrifuged at $10000 \mathrm{~g}$ for $5 \mathrm{~min}$. The absorbance of hemoglobin in the supernatant was measured using a spectrophotometer at $540 \mathrm{~nm}$. Blood mixed with PBS served as a negative control, while blood mixed with ultrapure water served as a positive control. Percentage hemolysis of RBCs was subsequently calculated according to

Hemolysis $(\%)=\left(\mathrm{Abs}_{\mathrm{NP}}-\mathrm{AbS}_{\mathrm{PBS}}\right) /\left(\mathrm{Abs}_{\mathrm{water}}-\mathrm{AbS}_{\mathrm{PBS}}\right) \times 100 \%$

where $\mathrm{Abs}_{\text {water }}, \mathrm{Abs}_{\mathrm{NP}}$ and $\mathrm{Abs}_{\mathrm{PBS}}$ represents the absorbances of the respective RBC suspensions at $540 \mathrm{~nm}$.

Blood clotting by the different magnetic nanoparticles was also evaluated, using a recalcification assay. ${ }^{27,28} 90 \mu \mathrm{L}$ of citrate dextrose supplemented whole blood was mixed with $10 \mu \mathrm{L}$ of magnetic nanoparticles $\left(10 \mathrm{mg} \mathrm{mL}^{-1}\right.$ in PBS) in a 96-wells plate and $10 \mu \mathrm{L}$ of $\mathrm{CaCl}_{2}$ solution (0.2 M in PBS) was added. After different time periods (5, 10, 15, 20 and $30 \mathrm{~min}$ ), $200 \mu \mathrm{L}$ of ultrapure water was gently added and $100 \mu \mathrm{L}$ of the suspension was transferred into a $1.5 \mathrm{~mL}$ centrifuge tube containing $900 \mu \mathrm{L}$ of ultrapure water and centrifuged at $10000 \mathrm{~g}$ for $5 \mathrm{~min}$. The absorbance of hemoglobin in the supernatant was measured using a spectrophotometer at $540 \mathrm{~nm}$. PBS without magnetic nanoparticles served as a control, represent- 
ing natural blood coagulation. The percentage blood coagulation was calculated as

$$
\begin{aligned}
\text { Blood coagulation }(\%)= & \left(\mathrm{Abs}_{0 \text { min }}-\mathrm{Abs}_{t \text { min }}\right) / \\
& \left(\mathrm{Abs}_{0 \text { min }}-\mathrm{Abs}_{\mathrm{PBS} 30 \text { min }}\right) \times 100 \%
\end{aligned}
$$

where $\mathrm{Abs}_{0}$ min and $\mathrm{Abs}_{t}$ min represent the absorbances of the suspension at $0 \mathrm{~min}$ and time t normalized to the absorbance of the control after $30 \mathrm{~min}$ in PBS $\left(\mathrm{AbS}_{\mathrm{PBS} 30} \mathrm{~min}\right)$. All experiments above were done in triplicate.

\section{In vivo eradication of a subcutaneous staphylococcal biofilm in mice}

For in vivo evaluation of the effects of artificial channel digging in an infectious biofilm on antibiotic efficacy, a subcutaneous staphylococcal infection was created in mice (Vital River Laboratory Animal Technology Co., Beijing, China). Experiments were performed in accordance with the guidelines of the Soochow University Laboratory Animal Center, China. Approval for our experiments by this committee was obtained 2020 May 22. Briefly, $100 \mu \mathrm{L}$ of $S$. aureus Xen36 $\left(10^{9}\right.$ CFU $\mathrm{mL}^{-1}$ in TSB) was injected subcutaneously into BALB/c nude mice (female, 7 weeks old, $18-20 \mathrm{~g}$ body weight). ${ }^{29}$ After $48 \mathrm{~h}$ of growth, mice were randomly assigned into four groups (6 mice in each group) and subjected to the following treatments: (a) $100 \mu \mathrm{L}$ of PBS, (b) $100 \mu \mathrm{L}$ of gentamicin $(10 \mathrm{mg}$ $\mathrm{mL}^{-1}$ yielding $50 \mathrm{mg}$ per $\mathrm{kg}$ body weight) in PBS, (c) $100 \mu \mathrm{L}$ of gentamicin $\left(10 \mathrm{mg} \mathrm{mL}^{-1}\right)$ in the presence of unmodified MIONPs (10 mg mL $\mathrm{mL}^{-1}$ ) in PBS and (d) $100 \mu \mathrm{L}$ gentamicin $\left(10 \mathrm{mg} \mathrm{mL} \mathrm{m}^{-1}\right)$ in the presence of unmodified MIONPs $(10 \mathrm{mg}$ $\mathrm{mL}^{-1}$ ) in PBS followed by magnetic propulsion for $10 \mathrm{~min}$. All injections were made locally underneath the infected area. For magnetic propulsion in group (d), a NdFeB magnet $(1 \mathrm{~mm}$ thickness and $10 \mathrm{~mm}$ in diameter with $1.2 \mathrm{~T}$ residual magnetism) was circled over the infectious area. Bioluminescent intensity was recorded once every two days from day 0 on, i.e. just before drug administration using a bio-optical imaging system (IVIS, $45 \mathrm{~s}$ exposure time, medium binning, $1 \mathrm{~F} / \mathrm{Stop}$, Open Emission Filter). Bioluminescent areas were calculated from the bioluminescent images using ImageJ software. Body weights of the mice were measured daily.

Immediately prior to sacrifice at day 8 , blood was collected from three mice of each group for blood analysis, including levels of alanine aminotransferase (ALT), aspartate aminotransferase (AST) and urea nitrogen (BUN) to detect possible influences of the treatments on liver and kidney function. Also, levels of white blood cells (WBC), red blood cells (RBC), platelets (PLT), hemoglobin (Hgb), hematocrit (HCT), mean corpuscular volume (MCV), mean corpuscular hemoglobin concentration (MCHC), and mean corpuscular hemoglobin $(\mathrm{MCH})$ were measured. Infected tissue was excised from the mice at sacrifice for CFU enumeration. Excised tissue was weighted, homogenized and suspended in $2 \mathrm{~mL}$ PBS, serially diluted and plated on TSB-agar with kanamycin for growth of $S$. aureus Xen36 and infection of excised tissue expressed as the number of CFUs retrieved per unit tissue weight. Excised tissue of one mouse from each group was immersed in 5\% formaldehyde for hematoxylin and eosin staining and histological analysis.

\section{Results and discussion}

\section{Preparation of MIONPs and PDA-modified MIONPs and their characterization}

Magnetic iron oxide nanoparticles were prepared using a hydrothermal method ${ }^{22}$ and PDA-modified ${ }^{30,31}$ as outlined in Fig. 1a. Zeta potentials of the nanoparticles were measured at $\mathrm{pH}$ 7.4, representing physiological conditions, at more acidic $\mathrm{pH}\left(\mathrm{pH}\right.$ 5.0) as relevant in a biofilm ${ }^{4,32}$ and in growth medium, containing high concentrations of proteins ${ }^{33,34}$ as also occurring in physiological body fluids. Zeta potentials of unmodified MIONPs were highly negative both in PBS as well as in TSB, regardless of $\mathrm{pH}$ (Fig. 1b). Zeta potentials became less negative upon PDA-modification. This attests to the exposure of cationic amino groups on PDA-modified MIONP surfaces, while phenolic hydroxyl groups of PDA are consumed in hydrogen bonding to the MIONP surfaces. ${ }^{35,36}$ Hydrodynamic diameters of unmodified MIONPs amounted $211 \mathrm{~nm}$, with a full width at half maximum of the distribution equal to $90 \mathrm{~nm}$ (Fig. 1c). PDA-modification slightly increased the hydrodynamic diameter of the MIONPs, but this was not statistically significant. Microscopically, the PDA layer appeared as a thin, translucent film around the nanoparticles, in correspondence with their slight increase in hydrodynamic diameter upon modification (Fig. 1d).

\section{Interaction between magnetic nanoparticles with staphylococci and EPS}

Two staphylococcal strains were used with different ability to produce EPS (EPS-producing $S$. aureus Xen36 and $S$. aureus 5298, producing far less EPS). ${ }^{18,19}$ Both strains showed negative zeta potentials in PBS and TSB, regardless of $\mathrm{pH}$ and whether suspended in PBS or TSB (Fig. 2a). Accordingly, the interaction of both strains with highly negatively-charged unmodified MIONPs (Fig. 1b) was low (Fig. 2b) in line with literature, ${ }^{17}$ due to strong electrostatic double-layer repulsion between staphylococci and unmodified MIONPs. Staphylococci and unmodified MIONPs remained well suspended without aggregation over a 5 min time-period (Fig. 2d). PDA-modified MIONPs were significantly less negativelycharged than unmodified MIONPs (Fig. 1b), particularly at $\mathrm{pH}$ 5, representing the acidic conditions as prevailing in a biofilm. ${ }^{37}$ Chemically, PDA is well-known as a bioadhesive ${ }^{26}$ and the decreases in electrostatic double-layer repulsion between PDA-modified MIONPs and negatively-charged staphylococci, next to ubiquitously present Lifshitz-van der Waals attraction, explains this adhesiveness on a physico-chemical basis (Fig. 2b) as compared with unmodified MIONPs. Due to lower electrostatic double-layer repulsion, staphylococci and PDA-modified MIONPs aggregated in suspension (Fig. 2d). Neither type of magnetic nanoparticles had any interaction with EPS (Fig. 2c). 
(a)

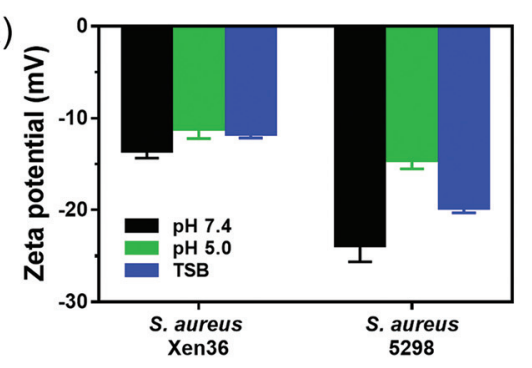

(b)

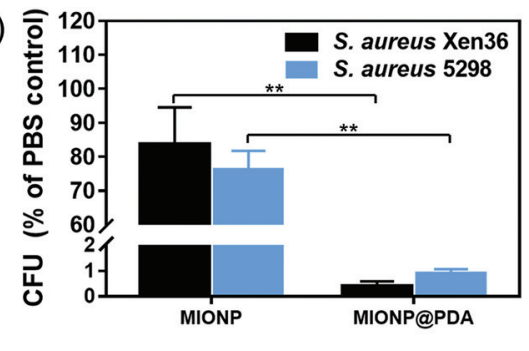

(c)

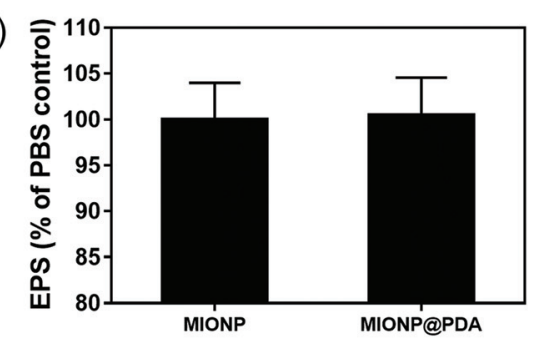

(d)
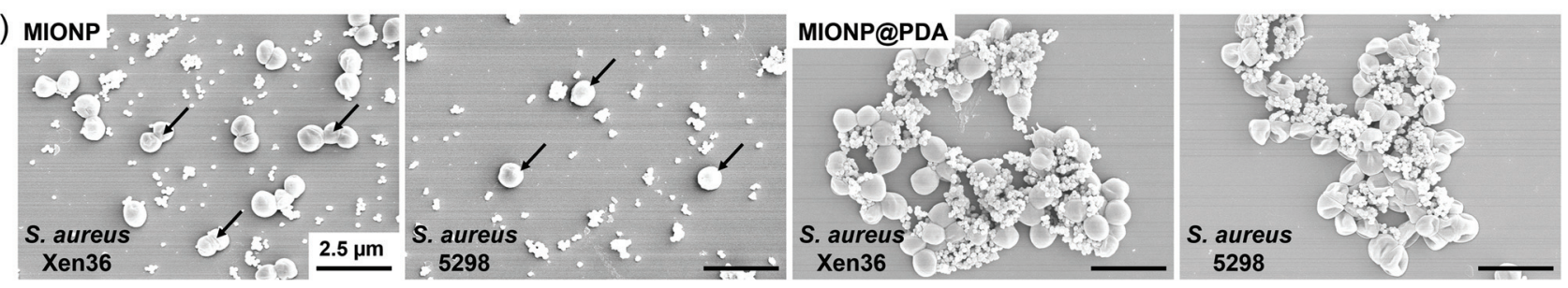

Fig. 2 Zeta potentials of the S. aureus strains used and the interaction between unmodified and PDA-modified magnetic nanoparticles with staphylococci or EPS. (a) Zeta potentials of S. aureus strains suspended in PBS (pH 7.4 and 5.0) and TSB. Zeta potentials are expressed as means \pm standard deviations over three separate experiments with differently cultured bacteria. (b) Percentage of CFUs of $S$. aureus suspended in PBS after mixing with unmodified or PDA-modified magnetic nanoparticles $\left(0.5 \mathrm{mg} \mathrm{mL}^{-1}\right)$ for $5 \mathrm{~min}$, followed by magnetic separation. The initial concentration of S. aureus suspended in PBS $\left(1 \times 10^{8} \mathrm{CFU} \mathrm{mL}{ }^{-1}\right)$ was set at $100 \%$. Data are expressed as means \pm standard deviations over three separate experiments with differently cultured bacteria. Statistical analysis was performed using the Student's two-tailed $t$-test $\left({ }^{* *} p<0.01\right)$. (c) Percentage of EPS left in solution after exposure to unmodified or PDA-modified MIONPs $\left(0.5 \mathrm{mg} \mathrm{mL}^{-1}\right)$ and magnetic separation. The weight of EPS left in solution after exposure to PBS without EPS was set at $100 \%$. Data are expressed as means \pm standard deviations over three separate experiments. (d) SEM micrographs showing the effect of exposing planktonic staphylococci suspended in PBS to unmodified and PDA-modified magnetic nanoparticles, under the conditions described for panel (b). Arrows point to bacteria.

\section{Efficacy of gentamicin in staphylococcal biofilms after additional artificial channel digging}

In order to explore whether the interaction of unmodified and PDA-modified MIONPs with bacteria or EPS played a role in channel digging and effects on antibiotic efficacy, gentamicin efficacy was evaluated with respect to bacterial killing with additional channels dug by unmodified and PDA-modified MIONPs. First, it was established that unmodified and PDAmodified MIONPs were not bactericidal (Table 1). Both strains were susceptible to gentamicin (see also Table 1), an aminoglycoside with a wide spectrum antibacterial efficacy, used mainly for local application.

Next, magnetic nanoparticles were magnetically propelled through staphylococcal biofilms parallel and perpendicular to

Table 1 Minimal inhibitory (MIC) and minimal bactericidal (MBC) concentrations of gentamicin and unmodified and PDA-modified MIONPs for S. aureus Xen36 and S. aureus 5298

\begin{tabular}{|c|c|c|c|c|}
\hline \multirow[b]{2}{*}{$\begin{array}{l}\text { MIC/MBC } \\
\text { against }\end{array}$} & \multicolumn{2}{|c|}{ S. aureus Xen36 } & \multicolumn{2}{|c|}{ S. aureus 5298} \\
\hline & $\begin{array}{l}\text { MIC } \\
\left(\mu \mathrm{g} \mathrm{mL}^{-1}\right)\end{array}$ & $\begin{array}{l}\mathrm{MBC} \\
\left(\mu \mathrm{g} \mathrm{mL}^{-1}\right)\end{array}$ & $\begin{array}{l}\text { MIC } \\
\left(\mu \mathrm{g} \mathrm{mL}^{-1}\right)\end{array}$ & $\begin{array}{l}\mathrm{MBC} \\
\left(\mu \mathrm{g} \mathrm{mL}^{-1}\right)\end{array}$ \\
\hline $\begin{array}{l}\text { Gentamicin } \\
\text { MIONP } \\
\text { MIONP@PDA }\end{array}$ & $\begin{array}{l}0.26 \pm 0.10 \\
>1000 \\
>1000\end{array}$ & $1.05 \pm 0.44$ & $\begin{array}{l}0.12 \pm 0.03 \\
>1000 \\
>1000\end{array}$ & $0.36 \pm 0.04$ \\
\hline
\end{tabular}

Data are expressed as mean \pm standard deviation over three experiments with separately cultured bacteria. the bottom of a well, after which biofilms were exposed to gentamicin $\left(1.25 \mathrm{mg} \mathrm{mL}^{-1}\right)$ for $3 \mathrm{~h}$. Exposure to gentamicin in absence of MIONPs killed $48 \%$ of EPS producing staphylococci which was significantly less killing than observed for the nonEPS producing strain (Fig. 3a and b), in line with the known protective effects of EPS production. ${ }^{38}$ Presence of both unmodified or PDA-modified MIONPs without magnetic propulsion did not affect staphylococcal killing in a biofilm-mode of growth, as also concluded from MIC and MBC measurements on planktonic staphylococci (Table 1). Artificial channel digging by magnetically propelled, non-interacting, unmodified MIONPs and exposure to gentamicin yielded larger killing than without magnetic propulsion (Fig. 3a and b). Interacting, PDA-modified MIONPs yielded significantly smaller enhancement of gentamicin killing than unmodified MIONPs. Enhanced killing in presence of artificial channel digging in biofilms of EPS producing $S$. aureus Xen36 by gentamicin was larger (Fig. 3a) than observed for the non-EPS producing strain (Fig. 3b). This is probably due to more effective channel digging by magnetically-propelled non-interacting MIONPs. This suggestion is confirmed in CLSM micrographs of staphylococcal biofilms after magnetic channel digging using noninteracting MIONPs, showing smaller, more uniform aggregates of $S$. aureus Xen36 bacteria (Fig. 4a) than when propelled through a non-EPS producing $S$. aureus 5298 biofilm, leaving aggregates amidst increased channelization (Fig. 4b).

Collectively, these findings demonstrate that artificial channel digging with beneficial effects on gentamicin penetration and killing requires propulsion of magnetic nano- 
(a)

S. aureus Xen36 (EPS producing strain)

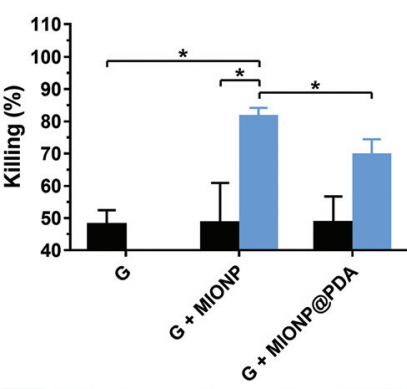

Without magnetic propulsion With magnetic propulsion

Fig. 3 Effects of magnetic channel digging by unmodified and PDAmodified MIONPs on the efficacy of subsequent exposure of staphylococcal biofilms to gentamicin (G). (a) Percentage killing after exposure of $S$. aureus Xen36 biofilms to magnetic nanoparticles $\left(0.5 \mathrm{mg} \mathrm{mL}^{-1}\right)$ with or without magnetic movement, followed by exposure to gentamicin $\left(1.25 \mathrm{mg} \mathrm{mL}^{-1}\right)$ for $3 \mathrm{~h}$ at $37^{\circ} \mathrm{C}$. Percentage killing was calculated with respect to the number of CFUs in biofilms exposed to PBS in the absence of both gentamicin and magnetic nanoparticles. (b) Similar to panel (a), now for $S$. aureus 5298 biofilms Data are expressed as means \pm standard deviations over three separate experiments with differently cultured bacteria. Statistical analysis was performed using the Student's two-tailed $t$-test $\left({ }^{*} p<0.05\right)$.

particles that do not interact with biofilm components. Since neither unmodified nor PDA-modified MIONPs interact with EPS, interaction of PDA-modified MIONPs with bacteria must be responsible for yielding a reduced enhancement of gentamicin efficacy as compared with unmodified MIONPs. This also follows from the aggregation of staphylococci in presence of PDA-modified MIONPs (Fig. 2d), which presumably led to collapse and clogging of channels by aggregates leading to a highly massive, dense biofilm (see CLSM micrographs in Fig. 4).

Efficacy of gentamicin against a sub-cutaneous staphylococcal biofilm in mice after additional, artificial channel digging by non-interacting, unmodified magnetic nanoparticles

Enhanced antibiotic efficacy against infectious biofilms after additional, artificial channel digging has never been explored in vivo to the best of our knowledge. Therefore, we here aimed to demonstrate in vivo benefits of artificial channel digging in $S$. aureus Xen36 biofilms using bioluminescence imaging ${ }^{29}$ and CFU enumeration in a sub-cutaneous infection model in mice (see Fig. 5a for experimental set-up). However, before carrying out animal experiments, it was first established that magnetic nanoparticles were biocompatible in vitro. Neither growth of fibroblasts (Fig. S1a $\dagger$ ), nor hemolysis (Fig. S1b $\dagger$ ) or blood coagulation (Fig. S1c $\dagger$ ) were negatively impacted in vitro by unmodified or PDA-modified magnetic nanoparticles. Since channel digging using non-interacting, unmodified MIONPs yielded the largest enhancement of antibiotic efficacy in vitro, only unmodified MIONPs were used for in vivo proof of prin-

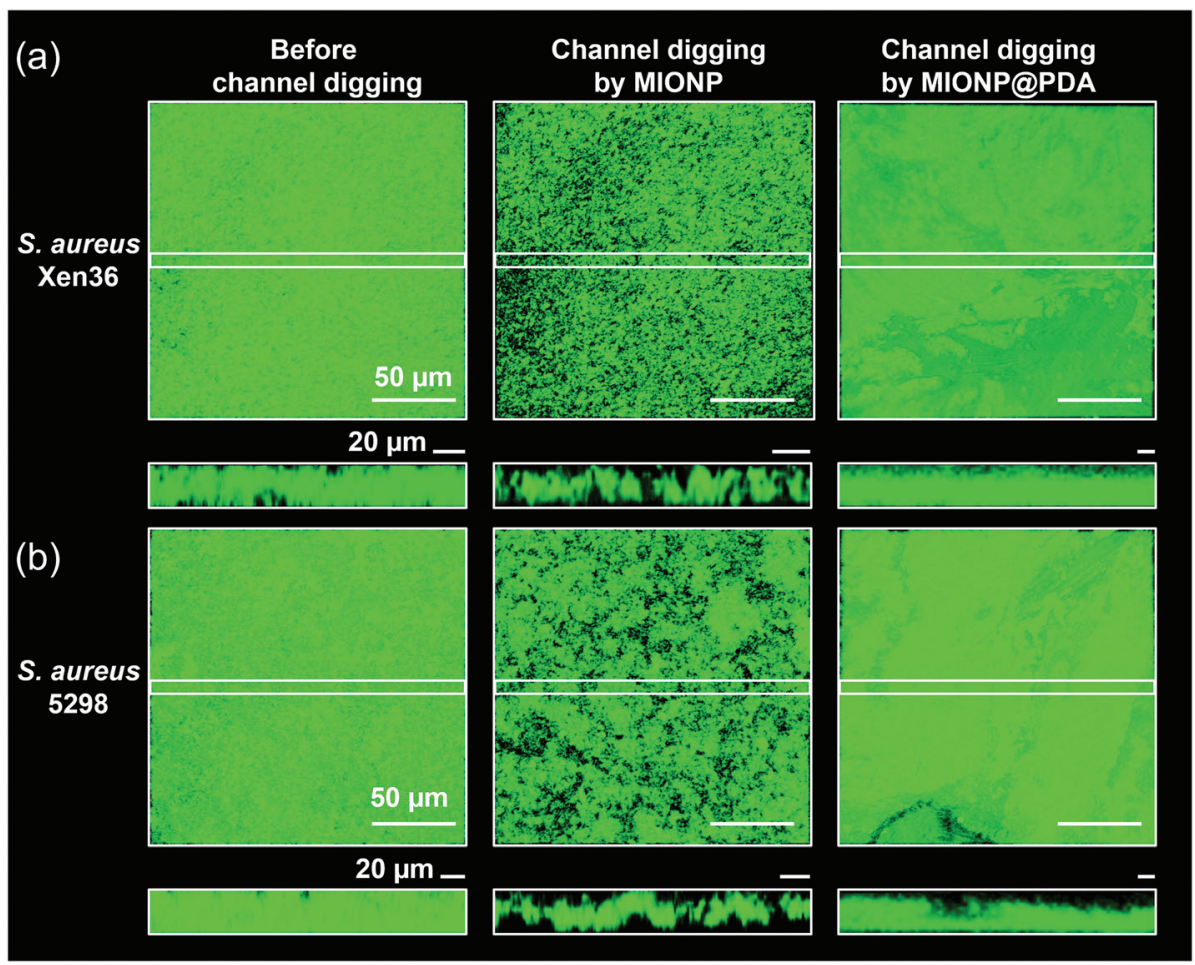

Fig. 4 CLSM micrographs of staphylococcal biofilms before and after magnetic channel digging by magnetic nanoparticles. (a) Overlayer and transverse, cross-sectional views of $24 \mathrm{~h}$, EPS producing S. aureus Xen36 biofilms prior to and after channel digging by magnetically-propelled non-interacting or interacting PDA-modified magnetic nanoparticles. Cross-sections were taken along the white line in the overlayer image. (b) Same as panel (a), now for EPS producing S. aureus 5298 biofilms. 
(a)

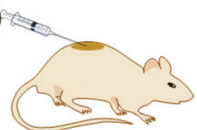

Sub-cutaneous s. aureus Xen 36 injection

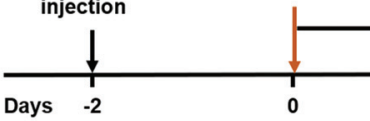

(b)
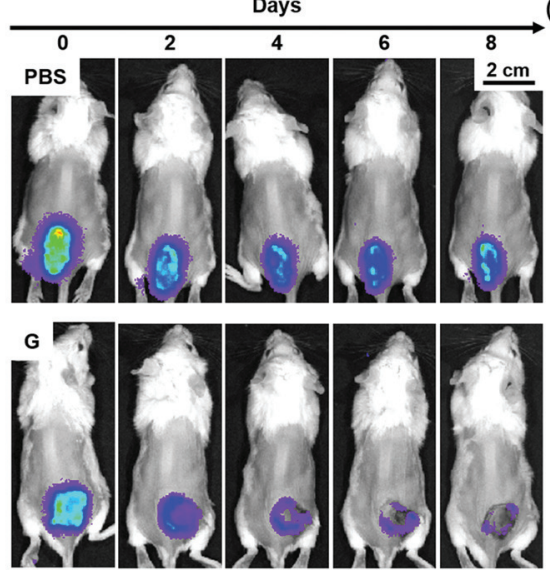

$G$ and MIONP
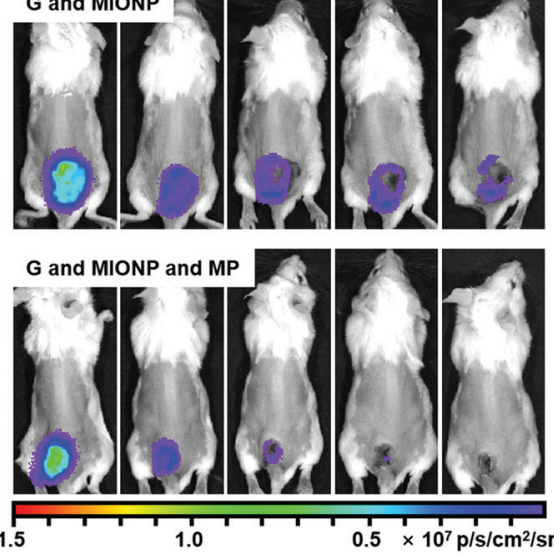

(f)
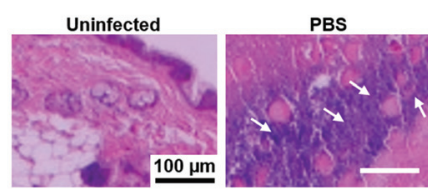

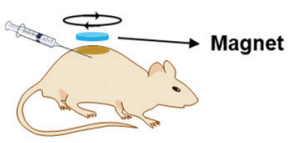

Drug Measure body weight daily

Image every other day Sacrifice

(c)

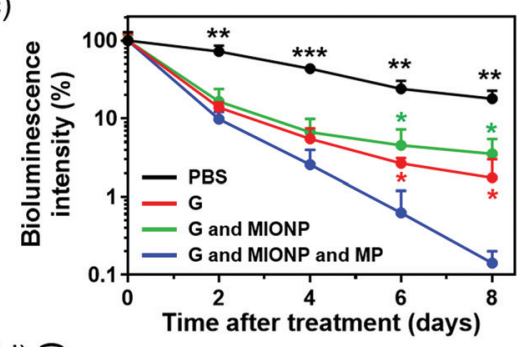

(d)

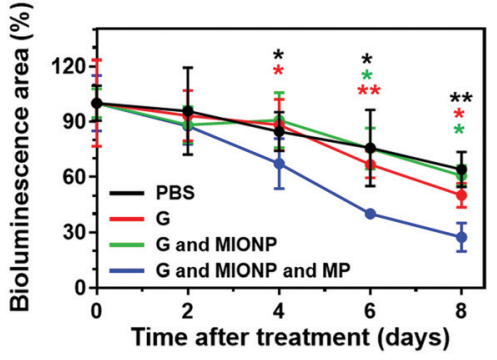

(e)
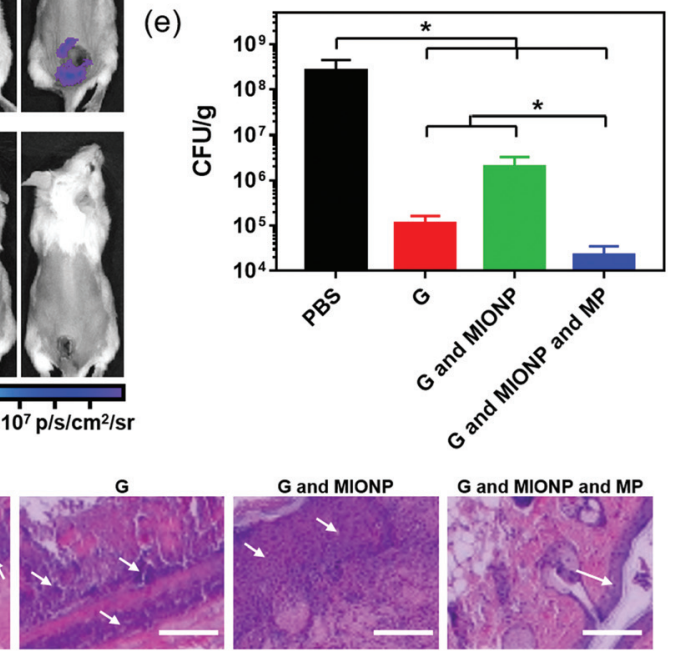

Fig. 5 Efficacy of gentamicin against sub-cutaneous infectious S. aureus Xen36 biofilms in absence and presence of additional, artificial channel digging by magnetically propelled, non-interacting, unmodified MIONPs in a murine model. (a) Time-scheme of the experiment. Mice were infected subcutaneously two days prior to drug administration at day 0 . Drug administration included four groups of mice: (i) PBS (100 $\mu \mathrm{L}$ ), (ii) gentamicin $\left(100 \mu \mathrm{L}, 10 \mathrm{mg} \mathrm{mL}^{-1}\right.$, i.e. roughly $50 \mathrm{mg}$ per kg body weight), (iii) gentamicin (G) and MIONPs (100 $\mu \mathrm{L}, 10 \mathrm{mg} \mathrm{mL}^{-1}$ of both G and MIONPs), (iv) G and MIONPs followed by magnetically-driven propulsion on MIONPs. Magnetic propulsion (MP) was only applied at day 0 , immediately after administration of MIONPs by circular movement of the magnet above the infection site for $10 \mathrm{~min}$. (b) Examples of bioluminescence images over time in the same mouse of each of the groups described in panel (a). (c) Relative bioluminescence intensity of each of the groups described in panel (a) as a function of time after drug administration. $100 \%$ bioluminescence intensity indicates the average intensity of all mice at day 0 prior to drug administration. (d) Same as panel c, now for the bioluminescence area. (e) Same as panel c, now for CFU $\mathrm{g}^{-1}$ in tissue excised from the infection site at sacrifice. (f) Histological analyses of tissue excised from the infection site after hematoxylin and eosin (H\&E) staining. White arrows indicate inflamed tissue. All quantitative data in this figure are expressed as means \pm standard deviations over four mice in each group. * indicate statistical significance levels (two-tailed Student's $t$-test) of the differences between groups $\left({ }^{*} p<0.05,{ }^{* *} p<0.01,{ }^{* * *} p<0.001\right.$ ). In panels $c$ and d, different colors refer to a comparison of the correspondingly colored group with the group that was treated with gentamicin and magnetically-driven MIONP movement. 
ciple to spare animal lifes. ${ }^{39}$ Quantitative bioluminescence data were derived from bioluminescence images of the infection site (Fig. 5b). Bioluminescent intensity (Fig. 5c) as well as the bioluminescent area (Fig. 5d) decreased significantly faster in the group of mice that was treated with gentamicin and MIONPs followed by their magnetic propulsion than in the corresponding group in absence of magnetic MIONP propulsion. CFU enumeration in excised tissue surrounding the infection site (Fig. 5e), confirmed that MIONPs magnetically propelled through the biofilms yielded the largest CFU reduction, concurrent with a relatively low number of inflammatory cells in excised tissue surrounding the infection site (Fig. 5f). Importantly, unmodified MIONPs exerted no adverse effects on the body weight (Fig. S2a $\dagger$ ), blood chemistry and routine blood parameters ${ }^{40,41}$ of the mice at sacrifice (Fig. S2b-2i †).

\section{Conclusions}

This is the first paper to show that artificial channel digging by magnetically-propelled nanoparticles through an infectious biofilm enhances the efficacy of antibiotic treatment in vivo. This innovative use of magnetically propelling magnetic nanoparticles through a biofilm, instead of magnetically collecting nanoparticles in a biofilm, eliminates the need for precise magnetic targeting methods, that are currently unavailable at the micron-level. Yet, mechanisms of artificial channel digging are unclear, including the role of interaction between magnetic nanoparticles with biofilm components, i.e. bacteria and the EPS they produce. We here show that interaction between magnetically-propelled nanoparticles with biofilm bacteria is undesirable, as it led to aggregation during channel digging, yielding a dense biofilm impeding deep antibiotic penetration and killing. This advance in our understanding of the mechanism of artificial channel digging constitutes an important step towards developing optimizing the surface properties of magnetic nanoparticles for use in artificial channel digging and its clinical application for enhancing antibiotic efficacy in hardto-treat infectious biofilms in patients.

\section{Author contributions}

KQ: data curation, formal analysis, investigation, methodology, writing original draft, writing review and editing. GJ: data curation, formal analysis, investigation, methodology, writing original draft, writing review and editing. JL: project administration, resources, supervision. ZZ: funding acquisition, project administration, resources, supervision, writing review and editing. YR: funding acquisition, project administration, writing review and editing. HJB: conceptualization, funding acquisition, project administration, supervision, writing original draft, writing review and editing. HCvdM: conceptualization, project administration, supervision, writing review and editing. BWP: supervision, writing review and editing.

\section{Conflicts of interest}

HJB is also director of a consulting company, SASA BV (GN Schutterlaan 4, 9797 PC Thesinge, The Netherlands). The authors declare no potential conflicts of interest with respect to authorship and/or publication of this article. Opinions and assertions contained herein are those of the authors and are not construed as necessarily representing views of their respective employers.

\section{Acknowledgements}

This work was financially supported by Natural Science Foundation of the Jiangsu Higher Education Institutions of China (20KJA150008), the National Natural Science Foundation of China (21522404), and UMCG, Groningen, The Netherlands.

\section{References}

1 M. K. Suresh, R. Biswas and L. Biswas, Int. J. Med. Microbiol., 2019, 309, 1-12.

2 N. Barraud, M. Kelso, S. Rice and S. Kjelleberg, Curr. Pharm. Des., 2015, 21, 31-42.

3 T. K. Nguyen, H. T. T. Duong, R. Selvanayagam, C. Boyer and N. Barraud, Sci. Rep., 2015, 5, 18385.

4 D. Hu, Y. Deng, F. Jia, Q. Jin and J. Ji, ACS Nano, 2020, 14, 347-359.

5 Z. Yuan, C. Lin, Y. He, B. Tao, M. Chen, J. Zhang, P. Liu and K. Cai, ACS Nano, 2020, 14, 3546-3562.

6 L. H. Reddy, J. L. Arias, J. Nicolas and P. Couvreur, Chem. Rev., 2012, 112, 5818-5878.

7 K. Ulbrich, K. Holá, V. Šubr, A. Bakandritsos, J. Tuček and R. Zbořil, Chem. Rev., 2016, 116, 5338-5431.

8 C. Boyer, M. R. Whittaker, V. Bulmus, J. Liu and T. P. Davis, NPG Asia Mater., 2010, 2, 23-30.

9 M. H. Kim, IEEE Trans. Nanobioscience, 2016, 15, 294-304.

10 L. de A. S. de Toledo, H. C. Rosseto and M. L. Bruschi, Pharm. Dev. Technol., 2018, 23, 316-323.

11 K. Quan, Z. Zhang, Y. Ren, H. J. Busscher, H. C. van der Mei and B. W. Peterson, J. Mater. Sci. Technol., 2021, 69, 69-78.

12 K. Quan, Z. Zhang, Y. Ren, H. J. Busscher, H. C. Van Der Mei and B. W. Peterson, ACS Biomater. Sci. Eng., 2020, 6, 205-212.

13 B. Shapiro, S. Kulkarni, A. Nacev, S. Muro, P. Y. Stepanov and I. N. Weinberg, Wiley Interdiscip. Rev.: Nanomed. Nanobiotechnol., 2015, 7, 446-457.

14 B. Shapiro, S. Kulkarni, A. Nacev, A. Sarwar, D. Preciado and D. A. Depireux, Annu. Rev. Biomed. Eng., 2014, 16, 455481.

15 J. N. Wilking, V. Zaburdaev, M. De Volder, R. Losick, M. P. Brenner and D. A. Weitz, Proc. Natl. Acad. Sci. U. S. A., 2013, 110, 848-852. 
16 A. Houry, M. Gohar, J. Deschamps, E. Tischenko, S. Aymerich, A. Gruss and R. Briandet, Proc. Natl. Acad. Sci. U. S. A., 2012, 109, 13088-13093.

17 K. Quan, Z. Zhang, H. Chen, X. Ren, Y. Ren, B. W. Peterson, H. C. van der Mei and H. J. Busscher, Small, 2019, 15, 1902313.

18 H. N. Rasyid, H. C. Van Der Mei, H. W. Frijlink, S. Soegijoko, J. R. Van Horn, H. J. Busscher and D. Neut, Acta Orthop., 2009, 80, 508-513.

19 Y. Liu, H. J. Busscher, B. Zhao, Y. Li, Z. Zhang, H. C. van der Mei, Y. Ren and L. Shi, ACS Nano, 2016, 10, 4779-4789.

20 R. Liu, Y. Guo, G. Odusote, F. Qu and R. D. Priestley, ACS Appl. Mater. Interfaces, 2013, 5, 9167-9171.

21 W. Cheng, X. Zeng, H. Chen, Z. Li, W. Zeng, L. Mei and Y. Zhao, ACS Nano, 2019, 13, 8537-8565.

22 H. Lee, J. Rho and P. B. Messersmith, Adv. Mater., 2009, 21, 431.

23 W. Cheng, K. Tang, Y. Qi, J. Sheng and Z. Liu, J. Mater. Chem., 2010, 20, 1799-1805.

24 P. M. Bales, E. M. Renke, S. L. May, Y. Shen and D. C. Nelson, PLoS One, 2013, 8, e67950.

25 G. Jarockyte, E. Daugelaite, M. Stasys, U. Statkute, V. Poderys, T. C. Tseng, S. H. Hsu, V. Karabanovas and R. Rotomskis, Int. J. Mol. Sci., 2016, 17, 1193.

26 G. Li, K. Quan, Y. Liang, T. Li, Q. Yuan, L. Tao, Q. Xie and X. Wang, ACS Appl. Mater. Interfaces, 2016, 8, 35071-35080.

27 M. F. Shih, M. Da Shau, M. Y. Chang, S. K. Chiou, J. K. Chang and J. Y. Cherng, Int. J. Pharm., 2006, 327, 117125.

28 S. Y. Ong, J. Wu, S. M. Moochhala, M. H. Tan and J. Lu, Biomaterials, 2008, 29, 4323-4332.

29 Y. Liu, H. C. van der Mei, B. Zhao, Y. Zhai, T. Cheng, Y. Li, Z. Zhang, H. J. Busscher, Y. Ren and L. Shi, Adv. Funct. Mater., 2017, 27, 1701974.
30 N. N. M. Adnan, Z. Sadrearhami, A. Bagheri, T. K. Nguyen, E. H. H. Wong, K. K. K. Ho, M. Lim, N. Kumar and C. Boyer, Macromol. Rapid Commun., 2018, 39, 1-8.

31 C. Xu, K. Xu, H. Gu, R. Zheng, H. Liu, X. Zhang, Z. Guo and B. Xu, J. Am. Chem. Soc., 2004, 126, 9938-9939.

32 S. C. Dexter and P. Chandrasekaran, Biofouling, 2000, 15, 313-325.

33 S. J. Li, M. Peng, H. Li, B. S. Liu, C. Wang, J. R. Wu, Y. X. Li and R. Zeng, Nucleic Acids Res., 2009, 37, D907-D912.

$34 \mathrm{~W}$. van Oeveren, in Biomaterials in Modern Medicine: The Groningen Prospective, ed. G. Rakhorst and R. Ploeg, World Scientific Publishing Co., Singapore, 1st edn, 2008, ch. 5, pp. 67-92.

35 D. Yang, X. Kong, Y. Ni, M. Ruan, S. Huang, P. Shao, W. Guo and L. Zhang, Polymer, 2019, 11, 218.

36 Y. Yang, P. Qi, Y. Ding, M. F. Maitz, Z. Yang, Q. Tu, K. Xiong, Y. Leng and N. Huang, J. Mater. Chem. B, 2015, 3, 72-81.

37 J. M. Vroom, K. J. de Grauw, H. C. Gerritsen, D. J. Bradshaw, P. D. Marsh, G. K. Watson, J. J. Birmingham and C. Allison, Appl. Environ. Microbiol., 1999, 65, 3502-3511.

38 H.-C. Flemming and J. Wingender, Nat. Rev. Microbiol., 2010, 8, 623-633.

39 H. J. Busscher, W. Woudstra, T. G. van Kooten, P. Jutte, L. Shi, J. Liu, W. L. J. Hinrichs, H. W. Frijlink, R. Shi, J. Liu, J. Parvizi, S. Kates, V. M. Rotello, T. P. Schaer, D. Williams, D. W. Grainger and H. C. van der Mei, Biomaterials, 2020, 232, 119737.

40 W. Xiu, S. Gan, Q. Wen, Q. Qiu, S. Dai, H. Dong, Q. Li, L. Yuwen, L. Weng, Z. Teng, Y. Mou and L. Wang, Research, 2020, 2020, 1-15.

41 D. Zhong, W. Li, Y. Qi, J. He and M. Zhou, Adv. Funct. Mater., 2020, 30, 1910395. 